\title{
Suicide, under-employment and poverty: the gendered impacts of COVID-19 in Japan
}

Edition 5, 2021

Associate Professor Nana Oishi

DOI: 10.37839/MAR2652-550X5.11

Japan has so far been perceived as handling the COVID-19 pandemic relatively well. Although the number of newly recorded cases of infection has been soaring since it was hit by a 'third wave' in November, its death rate is still lower than most industrialised countries: the number of deaths per 100,000 population is 2.66 as of January 3, 2021, compared with Australia (3.64), France (95.85), Germany (41.58) and the United States (107.04). Despite its economic downturn, Japan's unemployment rate remained at 3.1 percent as of October 2020, which is less than half of the average (7.06 percent) of industrialised countries.

Nevertheless, the socio-economic impacts of COVID-19 have still been significant in Japan and affected the lives of many, particularly those of women. Analyses of data between February (when the first COVID-19 case was found in Japan) and October (the latest available data) found that the employment impact on women was not as grave as widely suspected.

However, the official employment data may not necessarily present an accurate picture as they seem to include many cases of underemployment-situations where individuals officially still hold positions, but may not be working at all or being paid. Increasing cases of suicide among women, as well as higher rates of sexual and domestic violence are alarming, suggesting the further deterioration of women's mental and physical well-being and the feminisation of poverty. 
As revealed in studies across many countries, COVID-19 has had adverse effects on women and girls worldwide, to the extent that the United Nations Secretary-General, António Guterres, made a special request to member states to 'put women and girls at the centre of efforts to recover from COVID-19.' The pandemic's gendered impacts are also prominent in Japan, which has long been known for its gender inequality.

\section{Employment}

Since the pandemic hit Japan, there have been many media reports on massive job losses-dubbed as 'korona kaiko (corona-related dismissal)'-principally for those who hold precarious 'irregular jobs' (hiseiki koyo). According to the Japanese government's labour survey data, women comprised approximately 70 percent of 'irregular workers' in precarious, low-paid jobs in February 2020. Consequently, women are far more vulnerable to the economic downturn triggered by the pandemic.

And yet, the reality is slightly more complex. Contrary to popular belief, the number of women in 'regular employment' (seiki koyo) increased by 460,000 (3.97 percent) between February and October 2020. This change is assumed to be due to a legal change: a law aimed at ensuring 'equal pay for equal work' which became effective in April 2020 required corporations to reclassify irregular employees as regular employees if they were performing identical tasks. Within a month, the number of women in 'regular employment' rose by 380,000 (3.26 percent), even though a state of emergency had been partially imposed. Although no specific data are available, it is fair to estimate that many of these women were formerly 'irregular workers'; in short, their status was converted to 'regular workers' due to this legal change.

The number of men in regular employment also increased, but only by 0.81 percent in April 2020, and declined later. Between February and October, 410,000 Japanese men lost their jobs (1.73 percent), which is in stark contrast with the increase of 
460,000 women (3.97 percent). In addition to legal implications, it is important to remember that men in 'regular employment,' particularly those in senior positions, are likely to be exposed to companies' pandemic-related cost-cutting measures in the form of early retirement and voluntary redundancies.

In contrast, the situation of those in 'irregular employment,' such as casual workers, is quite different since they are not as protected as 'regular workers.' The pandemic affected women more significantly than men in this type of employment at least in the early stages when the state of emergency was declared. The drop in the number of women in irregular employment was particularly acute between March and April, reaching 940,000 (6.38 percent), in contrast with 360,000 (5.32 percent) of men. This drop is partly due to their status conversion to regular workers $(460,000)$ as discussed above, but the remaining number should be attributed to job losses and women's withdrawals from the labour market. Japanese schools and facilities were shut down in March even before the state of emergency was declared, and many families had to decide how to handle childcare. It is assumed that some women had to quit their jobs to assume this family responsibility since they tend to work under precarious conditions without benefits such as childcare leave.

\section{Gender inequality in unpaid work}

These data also underscore pre-existing gender inequality in unpaid work in Japan. In 2016, Japanese women spent 224 minutes a day on childcare, care of the elderly and household work, compared with 41 minutes spent by men, which was one of the greatest discrepancies seen in industrialised countries. During the pandemic, this situation has further deteriorated. According to a June 2020 survey, 37.8 percent of working women said they were spending more time on care work, and 30 percent on more housework than before the pandemic. In contrast, only 19.4 percent of men claimed the same for caretaking and 14 percent for housework.

Another survey showed that 78 percent of women reduced their amount of free time 
and 31 percent cut back on time spent on daily activities like bathing and sleeping to cope with more care and housework. The percentage of men who did the same was only 47 percent and 25 percent respectively. In both cases, a higher percentage of men than women were working from home, spending less time commuting and/or had reduced work-related hours. The extra time that men had available, however, did not necessarily translate into more time on caretaking or housework. These data imply that even among working couples in contemporary Japan, traditional gender norms are persistent, and women shoulder even greater burdens due to the pandemic.

\section{Increasing suicides}

The most concerning trend in Japan associated with the pandemic is a surge in suicides. While Japan has long been known for its high suicide rate, the number of suicide cases had been declining over the past 10 years, hitting a record low of 20,169 in 2019. Nevertheless, it spiked in March 2020 and has been rapidly increasing since then, despite a few slight downturns throughout the year. Overall, the number of suicides increased by a staggering 48.5 percent between February and October 2020.

Suicides have always been more common among men than women in Japan: the number of men's suicides had traditionally been twice as that of women. Nevertheless, what is unprecedented in the pandemic era is the accelerating rate of increase in female suicides. It rose by 100 percent between February and October 2020 , though there were a few downturns. This was in stark contrast with the increase in men's suicide (+27 percent) in the same period. The number of female suicides in October 2020 was 82.6 percent higher than the figure of the previous year. The percentage of women in the total suicide cases also rose from 29.3 percent in February to 39.5 percent in October. One study found that the number of suicides among young women was notably higher than in past years. 


\section{Underemployment and hidden poverty}

Although many reports attribute the increase in female suicides to job losses, this link does not seem so clear and straightforward. For instance, the biggest spike in female suicides (+30.0 percent) was observed between September and October, but women's employment increased during this period and also during the prior three months. Overall unemployment among women has also been relatively low: 2.7 percent in October, which is only a 0.5 percent increase from February 2020.

The possible keys to understanding female suicides could be underemployment and hidden poverty. This means that many workers technically remain 'employed' but suffer from a severe financial loss due to reduced working hours. As seen in other parts of the world, many Japanese enterprises shut down or shortened their operating hours while keeping their staff's positions.

There were 1.59 million workers who were temporarily put 'on leave' in April-June 2020, which was 10 times higher than the same period in the previous year, and 51.6 percent were women. The Japanese government offered these workers financial support of up to 330,000 yen (US\$3,210) a month, regardless of regular or irregular status. However, according to one media report, many small and medium-sized companies are not willing to apply for their employees' benefits since the process is too complex and time-consuming: there were only 12,857 applications submitted for this support between February and May, out of which only 5,052 cases were granted benefits. While the number of applications has been increasing since then, it is still not possible to fully grasp the situation as full data on the number of beneficiaries are not yet available. Nevertheless, it seems clear that even when workers officially hold their positions, keeping official unemployment rates low, a large number of them may be facing acute financial hardship, particularly if they have no access to financial support from the government.

Women are more likely to suffer from underemployment and poverty since they tend to be concentrated in precarious jobs in small and medium-sized companies. Since 
most women already earn significantly less than men, the lack of access to the government support could hit them harder than men.

It is worth noting that the number of welfare recipients has not increased between January and September 2020 despite the rise in unemployment and underemployment. Many studies have found that a sense of shame among the poor, societal stigma and strict gatekeeping by welfare officers often make welfare not easily accessible. Therefore, it is highly likely that the current welfare figures do not necessarily represent the real situations of unemployment, underemployment and poverty in Japan.

\section{Violence against women}

Growing violence against women during the pandemic is becoming a global issue-it has been observed across the world and is referred to by the United Nations as a 'shadow pandemic.' This has become a serious concern for Japan as well. The reports of sexual crime and violence, most of which are committed against women, rose by 15.5 percent in April-September 2020, compared with the same period in 2019. In particular, the number of calls related to domestic violence to government and municipal offices in May and June 2020 was 60 percent higher than the same month in the previous year. While gender-specific data are not yet available, given that women comprised 80.3 percent of those who called the domestic violence consultation centre in 2019 , it is highly likely that the majority of the callers (and the victims) are women.

Most domestic violence victims have been trapped with their abusive partners and have few options to change their situation due to the temporary shutdown of shelters and the suspension of many public services and social assistance from nongovernmental organisations. Even if women are able to escape their abusive partners, they face economic hardship because of poorer job prospects. They also have difficulty accessing the government's pandemic emergency package of 100,000 
yen (US\$973) per individual, which is deposited into the bank account of a household head, which is usually a husband. In cases where women escaped their husbands without officially changing their residential registry, they cannot obtain access to the support package.

A report by the Japan Suicide Countermeasures Promotion Center suggests that domestic violence can be one of the factors behind the increase in women's suicide. Its data showed that women who lived with someone (whether a family member or non-family member) a significantly higher suicide rate. While more detailed data are needed to determine whether they were the victims of domestic violence, the experts implied that this was the case.

\section{Well-being of youth and children}

As many countries report that children and young people tend to be more vulnerable to mental health issues than other age groups, future research also needs to address the pandemic impact on the well-being of youth and children. The number of calls to child support centres concerning abuse cases hit a record high of 194,000 in 2019, and the latest data show that it continued to increase even at a higher pace in January-August 2020. Given that domestic violence against women also signifies psychological abuse against their child/children, increasing cases of direct and indirect abuse against women are likely to exert a major impact on the mental wellbeing of Japan's youth.

Growing youth suicides also need more attention as they comprise a significant portion of the increasing suicide cases in Japan. One study found that the increase has been not just among those in middle and high schools: the increase among university students almost doubled in August and September 2020 compared with the average in 2017-2019 in each month. Further analyses of the gendered impacts on young people are essential. 


\section{Protections for the most vulnerable}

As the pandemic tends to hit the most socially vulnerable groups hardest across the world, targeted social protection measures are needed. In Japan, these groups include single mothers and their children who had already been suffering from severe poverty. Japan has the highest poverty rate of single-parent families (56 percent) and the highest share of single-mothers in the labour force of all industrialised countries. Given that 'single-parent families' include those headed by single-fathers who earn almost twice as much as single-mothers, the poverty rate among single-mother households is estimated to be considerably higher. Indeed, the average annual income of single mothers is only 2.4 million yen per annum (US $\$ 23,343$ ) and the vast majority of them work in precarious low-paid jobs. While the government provided a special benefit package for single-parent households, it is a single lump sum of only 100,000 yen (US\$973).

The NGO report discussed above expressed concern in relation to the pandemic's impact on children in single-parent households and other low-income households, since many of their parents were in irregular employment and lost their jobs or were forced to reduce their working hours. These children are suffering from economic hardship and challenges in education, as many of them cannot access the devices and/or internet connections necessary for online learning.

\section{Toward a more inclusive post-pandemic society}

As observed in many other countries, the pandemic impacts on Japan have also been highly gendered, which reflect its pre-existing gender inequality. Despite the Japanese government's 'womenomics' policy that has sought to promote women's employment and career development over the last seven years, significant gender inequality remains, with Japan's 'gender gap' being ranked among the worst in the 
world (121 $1^{\text {st }}$ in 153 countries).

Persistent traditional gender norms, structural barriers and institutional policies had long placed many women in precarious employment and poverty, which have become aggravated since the pandemic hit the country. While the change to the law relating to equity in employment status and pay in April improved this situation to a certain extent, women who were stuck in irregular employment have been hit hard by the pandemic. The increase in the rate of women's suicide, domestic violence and sexual crime against women is also alarming. It is encouraging that in September the government set up a special committee to investigate the impacts and the challenges of the pandemic on women. Its recommendations in November 2020 urged the administration of Prime Minister Yoshihide Suga to take action in eight priority areas, including domestic violence, sexual violence and support for single parents. Hopefully, urgent measures will be adopted to tackle these problems.

While gender-specific data in areas such as health, employment, suicide and sexual crime have enabled us to understand the gendered impacts of the pandemic, only limited data on ethnic, national or sexual minorities have been collected in Japan, and many other countries including Australia. It is important to inform the public of the differential impacts of the COVID-19 pandemic on these diverse groups. The perspective of intersectionality is crucial as well. For example, to what extent are migrant women suffering from the current economic downturn? We know, for instance, that some migrant women in Japan lost their jobs and had to work in the sex industry to earn enough money to return to their home countries. Migrants and minorities may be facing a higher unemployment rate and more severe economic difficulty. The pandemic's impact on the mental health of minorities should also be investigated, especially since a 2019 study pointed out that ethnic minorities in Japan had a higher suicide rate than the mainstream population.

Responses to the pandemic require social inclusion. Collecting health, employment and economic data on diverse groups would be an essential first step for Japan and elsewhere to achieve a better and more cohesive post-pandemic society. 
*The exchange rate of Japanese yen to US dollar is 0.0096 (05/01/2020).

Image: A women only train carriage in Japan. Credit: Bex Walton/Flickr.

Quarantine, masks and dis/ease: social discourses of COVID-19 in Japan and Korea 